\title{
ABEL-LIKE DIFFERENTIAL EQUATIONS WITH A UNIQUE LIMIT CYCLE
}

\author{
M.J. ÁLVAREZ, J.L. BRAVO, AND M. FERNÁNDEZ*
}

\begin{abstract}
For the family of scalar Abel-like equations $x^{\prime}=A(t) x^{n}+$ $b B(t) x^{m}$, where $A(t)=\sum_{l=1}^{k} a_{l} \sin ^{i_{l}}(t) \cos ^{j_{l}}(t), B(t)=\sin ^{i_{b}}(t) \cos ^{i_{b}}(t)$, $a_{l}, b \in \mathbb{R}, n, m, k, i_{l}, j_{l}, i_{b}, j_{b} \in \mathbb{Z}^{+}, n, m \geq 2$, and $k \geq 1$, we characterize the existence of non-trivial limit cycles (periodic solutions that are isolated in the set of periodic solutions different from the trivial $x(t) \equiv 0)$ in terms of $n, m, k, i_{l}, j_{l}, i_{b}, j_{b}$.
\end{abstract}

\section{INTRODUCTION}

Consider the generalized Abel equation

$$
x^{\prime}=\sum_{l=1}^{n} a_{l}(t) x^{l},
$$

where $a_{l}(t), 1 \leq l \leq n$ are trigonometric polynomials. We shall say that a periodic solution of (1.1) is a limit cycle if it is isolated in the set of all periodic solutions of (1.1), and that the origin of (1.1) is a centre if there exists a punctured neighbourhood of it such that every orbit contained in that neighbourhood is periodic.

The problem of bounding the number of limit cycles of (1.1) in terms only of $n$ turned out to be impossible since Lins Neto [16] proved that, for a fixed $n \geq 3$, there are functions $a_{l}(t)$ such that equation (1.1) has $k$ limit cycles for all $k$. Consequently, a new problem arose consisting of bounding the number of limit cycles of (1.1), but now depending on $n$ and on the degrees of $a_{l}(t)$, $1 \leq l \leq n$. This problem has been proposed by several authors, including C. Pugh and A. Lins Neto [16] and Yu. Ilyashenko [15]. Besides its own intrinsic interest, it is strongly related to Hilbert's 16th problem since some planar systems can be transformed into an equation of the form (1.1) (see $[16]$ for more details on the quadratic case and $[10,13,14]$ for other planar systems).

Nevertheless, bounding the number of limit cycles of equation (1.1) has turned out to be very hard, and even in the simple case $n=3, a_{1}(t) \equiv 0$,

2010 Mathematics Subject Classification. 34C25.

Key words and phrases. Periodic solutions; Abel equation.

* Corresponding author.

M.J.A. was partially supported by grants MTM2005-06098-C02-1 and 2005SGR-00550. J.L.B. and M.F. were partially supported by grant FEDER(UE) MTM2008-05460. 
and $a_{2}(t), a_{3}(t)$ of degree one, the problem is unsolved. There are only some partial results (see, for instance, $[3,6]$ ).

In general, the usual way to study this problem is to consider a certain family of generalized Abel equations. The Hilbert number of this family is the maximum of the number of limit cycles that the equations of the family can have.

This problem has been extensively studied, mainly for the classical Abel equation $x^{\prime}=A(t) x^{3}+B(t) x^{2}+C(t) x$, obtaining that when $A(t)$ or $B(t)$ have definite sign then the equation has at most three limit cycles (see $[12,18]$ ). These results have been extended to (1.1), usually imposing that some of the functions $a_{l}(t)$ do not change sign (see for instance $\left.[1,11,17,20]\right)$. For the case where all the $a_{l}$ may change sign, see $[6,7]$.

Thus, one of the aims of this paper is study the role of the trigonometric monomials in the generation of non-trivial limit cycles of a specific case of equation (1.1) assuming conditions only on the exponents of $\sin t, \cos t$, and $x$. In particular, for every $n, m \geq 2, k \in \mathbb{N}$, and $i_{l}, j_{l}, i_{b}, j_{b} \in \mathbb{Z}^{+}=\{0,1, \ldots\}$, $1 \leq l \leq k$, we study the families of generalized Abel equations of the form

$$
x^{\prime}=\left(\sum_{l=1}^{k} a_{l} \sin ^{i_{l}}(t) \cos ^{j_{l}}(t)\right) x^{n}+b \sin ^{i_{b}}(t) \cos ^{j_{b}}(t) x^{m}
$$

where $a_{1}, \ldots, a_{k}, b \in \mathbb{R}$.

Denote $\mathbf{i}=\left(i_{1}, \ldots, i_{k}, i_{b}\right)$ and $\mathbf{j}=\left(j_{1}, \ldots, j_{k}, j_{b}\right)$, and define the Hilbert number $\mathcal{H}(n, m, \mathbf{i}, \mathbf{j})$ of $(1.2)-\mathcal{H}$ when no confusion is possible - as the supremum (it could be infinite) over $\left(a_{1}, \ldots, a_{k}, b\right) \in \mathbb{R}^{k+1}$ of the number of limit cycles of (1.2).

With this definition, $\mathcal{H}=0$ if and only if every bounded solution of (1.2) is periodic for every $\left(a_{1}, \ldots, a_{k}, b\right)$, while $\mathcal{H}=1$ if and only if the solution $x(t) \equiv 0$ is the only periodic solution of $(1.2)$ for every $\left(a_{1}, \ldots, a_{k}, b\right)$, except for some $\left(a_{1}, \ldots, a_{k}, b\right)$ for which the origin is a centre.

To simplify the notation, let $A_{l}(t)=\sin ^{i_{l}}(t) \cos ^{j_{l}}(t), B(t)=\sin ^{i_{b}}(t) \cos ^{j_{b}}(t)$, and define the following sets

$$
\begin{array}{ll}
\mathcal{S}=\left\{\sin ^{i}(t) \cos ^{j}(t): i \text { odd }, j \text { even }\right\}, & \mathcal{E}=\left\{\sin ^{i}(t) \cos ^{j}(t): i, j \text { even }\right\}, \\
\mathcal{C}=\left\{\sin ^{i}(t) \cos ^{j}(t): i \text { even, } j \text { odd }\right\}, & \mathcal{O}=\left\{\sin ^{i}(t) \cos ^{j}(t): i, j \text { odd }\right\} .
\end{array}
$$

In $[2$, Theorem 3.3] we proved the following result:

Theorem 1.1. Consider the family of equations

$$
\begin{array}{r}
x^{\prime}=\left(a_{1} A_{1}(t)+a_{2} A_{2}(t)\right) x^{n}+b B(t) x^{m}, \\
\left(i_{1}-i_{2}\right)\left(j_{1}-j_{2}\right) \leq 0, \quad n, m \geq 2,
\end{array}
$$

where $a_{1}, a_{2}, b \in \mathbb{R}$. Then

(1) $\mathcal{H}=0$ if and only if $A_{1}, A_{2}, B \in \mathcal{S} \cup \mathcal{O}$, or $A_{1}, A_{2}, B \in \mathcal{C} \cup \mathcal{O}$.

(2) $\mathcal{H}=1$ if and only if one of the following statements holds: 
(a) There exists a unique l such that $A_{l} \in \mathcal{E}$, and (1.3) has no limit cycles for $a_{l}=0$, or $B \in \mathcal{E}$ and (1.3) has no limit cycles for $b=0$

(b) $\mathcal{H} \neq 0, A_{l} \notin \mathcal{E}$ for any $1 \leq l \leq 2, B \notin \mathcal{E}$, and neither $A_{1}, A_{2} \in$ $\mathcal{C}, B \in \mathcal{S}$, nor $A_{1}, A_{2} \in \mathcal{S}, B \in \mathcal{C}$.

The aim of this paper is to extend this result to the more general family (1.2). Hence, we will prove the following characterizations for the family of differential equations (1.2) to have $\mathcal{H}=0$ and $\mathcal{H}=1$.

Theorem 1.2. Consider the family of equations (1.2). Then

(1) $\mathcal{H}=0$ if and only if $A_{1}, \ldots, A_{k}, B \in \mathcal{S} \cup \mathcal{O}$, or $A_{1}, \ldots, A_{k}, B \in \mathcal{C} \cup \mathcal{O}$.

(2) $\mathcal{H}=1$ if and only if one of the following statements holds:

(a) There exists a unique l such that $A_{l} \in \mathcal{E}$, and (1.2) has no limit cycles for $a_{l}=0$, or $B \in \mathcal{E}$ and (1.2) has no limit cycles for $b=0$.

(b) $A_{1} \in \mathcal{S}, A_{2}, \ldots, A_{k} \in \mathcal{C} \cup \mathcal{O}$, and $B \in \mathcal{C}$, reordering the $A_{l}$, $1 \leq l \leq k$, if necessary.

(c) $A_{1} \in \mathcal{C}, A_{2}, \ldots, A_{k} \in \mathcal{S} \cup \mathcal{O}$, and $B \in \mathcal{S}$, reordering the $A_{l}$, $1 \leq l \leq k$, if necessary.

(d) $A_{1} \in \mathcal{S}, A_{2} \in \mathcal{C}, A_{3}, \ldots, A_{k} \in \mathcal{O}$, and $B \in \mathcal{O}$, reordering the $A_{l}, 1 \leq l \leq k$, if necessary. In the case $k=2$, this statement reduces to $A_{1} \in \mathcal{S}, A_{2} \in \mathcal{C}$, and $B \in \mathcal{O}$.

Theorem 1.2 is proved in the following sections. The proof will be split into two main parts.

First, in Section 2 we obtain upper bounds for the number of limit cycles. This covers the sufficiency on the two statements of the theorem. Concretely, Proposition 2.2 covers $(1),(2 a),(2 b)$, and $(2 c)$. Proposition 2.3 covers $(2 d)$. Moreover, this result extends the ones obtained in [7].

Second, in Section 4, for any of the remaining cases we obtain a limit cycle different from $x(t) \equiv 0$ by a Hopf bifurcation. This proves the necessary part of the two statements of the theorem. Proposition 4.1 proves that the remaining cases, reordering $\left\{A_{1}, \ldots, A_{k}\right\}$ if necessary, are:

(I.1) $A_{1}, A_{2} \in \mathcal{E}$.

(I.2) $A_{1}, B \in \mathcal{E}$.

(I.3) $A_{1} \in \mathcal{E}, A_{2} \in \mathcal{C}($ resp. $\mathcal{S}$ ), and $B \in \mathcal{S}$ (resp. $\mathcal{C}$ ).

(I.4) $A_{1} \in \mathcal{E}, A_{2} \in \mathcal{S}, A_{3} \in \mathcal{C}$, and $B \in \mathcal{O}$.

(I.5) $A_{1}, A_{2} \in \mathcal{C}$ (resp. $\mathcal{S}$ ) and $B \in \mathcal{S}$ (resp. $\mathcal{C}$ ).

(I.6) $A_{1} \in \mathcal{S}, A_{2} \in \mathcal{C}, A_{3} \in \mathcal{S} \cup \mathcal{C}$, and $B \in \mathcal{O}$.

Proposition 4.2 shows that in every of the cases above, one can get an additional limit cycle by a Hopf bifurcation of the solution $x(t) \equiv 0$.

Section 3 between the two parts will be devoted to obtaining some auxiliary results to compute the Hopf bifurcation using information provided by 
the moment problem. These new techniques have allowed to improve the results in [2], in particular, removing the technical condition $\left(i_{1}-i_{2}\right)\left(j_{1}-j_{2}\right) \leq$ 0 .

\section{UPPER BOUNDS ON THE NUMBER OF LIMIT CYCLES}

We begin this section with a definition that will be used recurrently throughout the paper.

Definition 2.1. Consider the differential equation $x^{\prime}=f(t, x)$. We say that a function $\alpha(t)$ is an upper-solution of this equation provided that $\alpha^{\prime}(t) \geq f(t, \alpha(t))$. We say that a function $\beta(t)$ is a lower-solution of this equation provided that $\beta^{\prime}(t) \leq f(t, \beta(t))$.

Most of the results we need on upper bounds are already proved in $[2,7]$. We shall recall these results and complete them with a new one.

The following proposition covers the results known already.

Proposition 2.2. Consider equation (1.2). Then

(1) $\left[2\right.$, Proposition 2.1]. If $A_{1}, \ldots, A_{k}, B \in \mathcal{S} \cup \mathcal{O}$, or $A_{1}, \ldots, A_{k}, B \in$ $\mathcal{C} \cup \mathcal{O}$, then (1.2) has a centre at $x=0$.

(2) $[2$, Proposition 2.2]. Assume that either there exists $l \in\{1, \ldots, k\}$ such that $A_{l} \in \mathcal{E}$ and if $a_{l}=0$ then (1.2) has a centre at $x(t) \equiv 0$ or that $B \in \mathcal{E}$ and if $b=0$ then (1.2) has a centre at $x(t) \equiv 0$. Then $x(t) \equiv 0$ is the only limit cycle of (1.2) for every $a_{l}, b \neq 0$.

(3) [7, Theorem 2.4] Suppose that $A_{1} \in \mathcal{S}$ (resp. $\left.A_{1} \in \mathcal{C}\right), A_{2}, \ldots, A_{k} \in$ $\mathcal{C} \cup \mathcal{O}$, and $B \in \mathcal{C}$ (resp. $A_{2}, \ldots, A_{k} \in \mathcal{S} \cup \mathcal{O}$, and $B \in \mathcal{S}$ ). Then $x(t) \equiv 0$ is the only possible limit cycle of (1.2). Moreover, for $a_{1}, \ldots, a_{k}, b \neq 0,(1.2)$ has no centre at $x(t) \equiv 0$.

(4) [7, Theorem 2.8] Suppose that $k=2, A_{1} \in \mathcal{S}$ (resp. $\left.A_{2} \in \mathcal{C}\right), A_{2} \in \mathcal{C}$ (resp. $\left.A_{2} \in \mathcal{S}\right), B \in \mathcal{O}$. Then $x(t) \equiv 0$ is the only possible limit cycle of (1.2).

Following the above proposition, the remaining case to be proved for the sufficiency of the conditions in Theorem 1.2 is the following.

Proposition 2.3. Suppose that $A_{1} \in \mathcal{S}, A_{2} \in \mathcal{C}, A_{3}, \ldots, A_{k} \in \mathcal{O}$, and $B \in \mathcal{O}$. Then $x(t) \equiv 0$ is the only limit cycle of $(1.2)$ for $a_{1} \neq 0, a_{2} \neq 0$, and $b \neq 0$. If $a_{1}, a_{2}$, or $b$ are null, then (1.2) has a centre at $x(t) \equiv 0$.

Proof. First, let us prove last sentence of the statement. If $a_{1}=0$ or $a_{2}=0$, then by Proposition 2.2(1), Eq. (1.2) has a centre at $x(t) \equiv 0$. If $b=0$, then (1.2) is an equation in separate variables, and by direct integration one obtains that (1.2) has a centre at $x(t) \equiv 0$.

The changes of variables $t \rightarrow-t, t \rightarrow \pi \pm t$ allow one to choose the signs of $a_{1}$ and $a_{2}$. Assume that $a_{1}, a_{2}<0$ and $(m-n) b>0$, with the case $(m-n) b<0$ being analogous. For brevity, we shall write $h(t)=$ $a_{3} A_{3}(t)+\ldots+a_{k} A_{k}(t)$. We recall that $h(t-k \pi / 2)$ is odd for every $k \in \mathbb{Z}$, since $A_{3}, A_{4}, \ldots A_{k} \in \mathcal{O}$. 
Consider the one-parameter family

$$
x^{\prime}=\left(a_{1} A_{1}(t)+\lambda a_{2} A_{2}(t)+h(t)\right) x^{n}+b B(t) x^{m}, \quad 0 \leq \lambda \leq 1 .
$$

For $\lambda=0,(2.4)$ has a centre at $x(t) \equiv 0$. We shall prove that $x(t) \equiv 0$ is the only periodic solution of (2.4) for $\lambda=1$.

Denote by $u\left(t, x, \lambda_{0}\right)$ the solution of (2.4) determined by $\lambda=\lambda_{0}$ and $u\left(0, x, \lambda_{0}\right)=x$. The proposition will be proved in two steps. In the first we shall prove that, if $u(t, x, \lambda)$ is defined for all $t \in[-\pi, \pi]$ and $\lambda \in[0,1]$ with $x>0$, then $u(-\pi, x, 1)>u(\pi, x, 1)$. In the second, we shall prove that, if $u(t, x, 1)$ is defined for all $t \in[-\pi, \pi]$, then $u(t, x, \lambda)$ is defined for all $t \in[-\pi, \pi]$ and $\lambda \in[0,1]$. The inequality $u(-\pi, x, 1)>u(\pi, x, 1)$ obtained in the first part shows that (2.4) has no positive periodic solutions for $\lambda=1$. By the change of variables $x \rightarrow-x$, we conclude that it has no negative periodic solutions. Thus, $x(t) \equiv 0$ is the only limit cycle.

Firstly, let us prove that, if $u(t, x, \lambda)$ is defined for all $t \in[-\pi, \pi]$ and $\lambda \in[0,1]$, then $u(-\pi, x, 1)>u(\pi, x, 1)$. In particular, we shall prove that for all $x>0$

$$
\frac{\partial u}{\partial \lambda}(\pi, x, \lambda)<0<\frac{\partial u}{\partial \lambda}(-\pi, x, \lambda) .
$$

Since $u(-\pi, x, 0)=u(\pi, x, 0)$ due to hypotheses, then $u(-\pi, x, 1)>u(\pi, x, 1)$.

By the change of variables $t \rightarrow-t$, and taking into account that $A_{1}, h, B$ are odd and $A_{2}$ is even,

$$
\frac{\partial u}{\partial \lambda}(-\pi, x, \lambda)=-\frac{\partial u}{\partial \lambda}(\pi, x,-\lambda) .
$$

Therefore, it is sufficient to prove that $\partial u(\pi, x, \lambda) / \partial \lambda<0$ for all $x>0$, $\lambda \in[-1,1]$.

Take $x>0, \lambda \in[-1,1]$, and denote $u(t)=u(t, x, \lambda)$. Deriving (2.4) with respect to $\lambda$ and integrating over $[0, \pi]$, one obtains

$$
\begin{aligned}
\frac{\partial u}{\partial \lambda}(\pi) & =\int_{0}^{\pi} a_{2} A_{2}(t) u^{n}(t) e^{\int_{t}^{\pi} n \frac{u^{\prime}}{u}+(m-n) b B(s) u^{m-1}(s) d s} d t \\
& =u^{n}(\pi) \int_{0}^{\pi} a_{2} A_{2}(t) e^{\int_{t}^{\pi}(m-n) b B(s) u^{m-1}(s) d s} d t .
\end{aligned}
$$

Since $u^{n}(\pi)>0$ and $A_{2}(t-\pi / 2)$ is odd, the sign of $\frac{\partial u}{\partial \lambda}(\pi)$ is the same as the sign of

$$
\int_{0}^{\pi / 2} a_{2} A_{2}(t)\left(e^{\int_{t}^{\pi}(m-n) b B(s) u^{m-1}(s) d s}-e^{\int_{\pi-t}^{\pi}(m-n) b B(s) u^{m-1}(s) d s}\right) d t .
$$

Now we shall prove that the expression in parentheses is positive for all $t \in(0, \pi / 2)$. Then, as $a_{2}<0$, it will follow that $\frac{\partial u}{\partial \lambda}(\pi)<0$.

This is equivalent to proving that

$$
\int_{t}^{\pi} B(s) u^{m-1}(s) d s-\int_{\pi-t}^{\pi} B(s) u^{m-1}(s) d s>0
$$


or equivalently

$$
\begin{aligned}
& \int_{t}^{\pi-t} B(s) u^{m-1}(s) d s \\
& =\int_{t}^{\pi / 2} B(s) u^{m-1}(s) d s+\int_{\pi / 2}^{\pi-t} B(s) u^{m-1}(s) d s>0 .
\end{aligned}
$$

As $B(t-\pi / 2)$ is odd, the above integrals can be rewritten as

$$
\int_{t}^{\pi / 2} B(s)\left(u^{m-1}(s)-u^{m-1}(\pi-s)\right) d s .
$$

To conclude, we shall prove that $u(s)>u(\pi-s)$ for all $s \in(0, \pi / 2)$. Recall that, due to the parities of $A_{l}$ and $B$, the function $u(\pi-t)$ is the solution of

$$
x^{\prime}=\left(-a_{1} A_{1}(t)+\lambda a_{2} A_{2}(t)+h(t)\right) x^{n}+b B(t) x^{m} .
$$

determined by $x(\pi / 2)=u(\pi / 2)$. Since $a_{1} A_{1}(t)+a_{1} A_{1}(\pi-t)=2 a_{1} A_{1}(t)<0$ for all $t \in(0, \pi / 2), u(\pi-t)$ is an upper-solution of (1.2) that coincides with $u(t)$ at $t=\pi / 2$. Therefore $u(t)>u(\pi-t)$ for all $t \in(0, \pi / 2)$ (note that reversing the time an upper-solution becomes a lower-solution).

Finally, we must prove that if $u(t, x, 1)$ is defined in $[-\pi, \pi]$, then $u(t, x, \lambda)$ is defined for all $t \in[-\pi, \pi], \lambda \in[0,1]$.

For all $\lambda \in[0,1], u(t, x, 1)$ is a lower-solution of $(2.4)$ in $\left(-\frac{\pi}{2}, 0\right)$. For each fixed $\lambda, u(-\pi-t, x, \lambda)$ is a lower-solution of $(2.4)$ in $\left(-\pi,-\frac{\pi}{2}\right)$ and $u(-t, x, \lambda)$ is an upper-solution of $(2.4)$ in $\left(0, \frac{\pi}{2}\right)$. Therefore $u(t, x, \lambda)$ is defined in $\left[-\pi, \frac{\pi}{2}\right]$. Moreover, since $u(t, x, 0)$ is symmetric with respect to $t=0$, it is defined in $[-\pi, \pi]$. Let us define

$$
I=\left\{\lambda_{0} \in[0,1]: u(t, x, \lambda) \leq u(t, x, 0), t \in\left[\frac{\pi}{2}, \pi\right], 0 \leq \lambda<\lambda_{0}\right\} .
$$

If $I=[0,1]$, then $0<u(t, x, \lambda) \leq u(t, x, 0)$, and $u(t, x, \lambda)$ is defined for all $t \in\left[\frac{\pi}{2}, \pi\right], \lambda \in I$.

We shall prove that $I$ is open and closed, and since $0 \in I$ then $I=[0,1]$.

If $\lambda_{0} \in I$, then by continuity of the solutions with respect to the parameters, $u(t, x, \lambda)$ is defined in $\left[\frac{\pi}{2}, \pi\right]$ for all $\lambda>\lambda_{0}$ close enough, and, since $\frac{\partial u}{\partial \lambda}(\pi, x, \lambda)<0, u(\pi, x, \lambda) \leq u\left(\pi, x, \lambda_{0}\right) \leq u(\pi, x, 0)$ for all $\lambda>\lambda_{0}$ close enough. Since $u(t, x, 0)$ is a lower-solution of $(2.4)$ in $\left[\frac{\pi}{2}, \pi\right]$, one obtains $u(t, x, \lambda) \leq u(t, x, 0)$ for all $t \in\left[\frac{\pi}{2}, \pi\right]$. Therefore $\lambda \in I$ and $I$ is open.

Let $\lambda_{n} \rightarrow \lambda_{0}, n \rightarrow \infty, \lambda_{n} \in I$. For all $0 \leq \lambda<\lambda_{0}$ and $t$ belonging to the interval of definition of $u\left(t, x, \lambda_{0}\right)$, one has $\lambda<\lambda_{n}$, for some $n$ large enough. Since $\lambda_{n} \in I, u(t, x, \lambda) \leq u(t, x, 0)$. Therefore $\lambda_{0} \in I$ and $I$ is closed. Consequently, $I=[0,1]$ and $u(t, x, \lambda)$ is defined for all $t \in[-\pi, \pi]$ and all $\lambda \in[0,1]$, concluding the proof. 


\section{Hopf Bifurcation and the Moment Problem}

In this section we introduce the basic notation and results for the Hopf bifurcations that we will need in the following final section to generate limit cycles for equation (1.2).

If $u\left(t, x, a_{1}, \ldots, a_{k}, b\right)$ is the solution of (1.2) determined by the initial condition $u\left(0, x, a_{1}, \ldots, a_{k}, b\right)=x$, then

$$
u\left(t, x, a_{1}, \ldots, a_{k}, b\right)=\sum_{j=0}^{\infty} v_{j}\left(t, a_{1}, \ldots, a_{k}, b\right) x^{j} .
$$

The following Lemma describes the Hopf bifurcations we shall be using.

Lemma 3.1 ([2, Lemma 3.2]). Consider Eq. (1.2). Let $a_{1}, a_{2}, \ldots, a_{k}, b \in \mathbb{R}$, and $j^{*}>1$ be such that the following conditions hold:

(i) $v_{j}\left(2 \pi, a, a_{2}, \ldots, a_{k}, b\right)=0$ for every $1<j<j^{*}$ and every $a \in \mathbb{R}$.

(ii) $v_{j^{*}}\left(2 \pi, \underline{a}_{1}, a_{2}, \ldots, b\right)<0<v_{j^{*}}\left(2 \pi, \bar{a}_{1}, a_{2}, \ldots, b\right)$ for every $\underline{a}_{1}<a_{1}<$ $\bar{a}_{1}$.

(iii) $x(t) \equiv 0$ is a limit cycle of (1.2) for $a_{1}, a_{2}, \ldots, a_{k}, b$.

Then there exists $a_{1}^{*} \in \mathbb{R}$ such that Eq. (1.2) has a positive limit cycle for $a_{1}^{*}, a_{2}, \ldots, a_{k}, b$.

The same result holds replacing $\underline{a}_{1}<a_{1}<\bar{a}_{1}$ by $\underline{a}_{1}>a_{1}>\bar{a}_{1}$. The result is also valid if we replace the role played by $a_{1}$ by $a_{l}, l=2, \ldots, k$ or by $b$.

The structure of the coefficients $v_{j}(t)=v_{j}\left(t, a_{1}, \ldots, a_{k}, b\right)$ is determined by the following result of [2, Appendix A], in which we set $A=\sum_{l=1}^{k} A_{l}$, $n_{a}=n$. Before stating this result, we shall define some notation. For any functions $C, D, E$, we denote

$$
\begin{gathered}
I_{C}(t)=\int_{0}^{t} C(s) d s, \quad I_{C D}(t)=\int_{0}^{t} C(s) \int_{0}^{s} D(u) d u d s, \\
I_{C D E}(t)=\int_{0}^{t} C(s) \int_{0}^{s} D(u) \int_{0}^{u} E(\sigma) d \sigma d u d s .
\end{gathered}
$$

Lemma 3.2 ([2, Proposition A.1]). There exist continuous functions $K_{k_{1}, k_{2}}(t)$ such that

$$
v_{j}(t)=\sum_{\substack{k_{1}, k_{2} \in \mathbb{Z}^{+} \\ j_{k_{1}}, k_{2}=j}} K_{k_{1}, k_{2}}(t),
$$

where

$$
j_{k_{1}, k_{2}}=k_{1} n+k_{2} m-\left(k_{1}+k_{2}-1\right) .
$$

In particular, for $k_{1}=k_{2}=0$, one has $v_{1}(t)=K_{0,0}(t) \equiv 1$. Thus every solution of $(1.2)$ defined in $[0,2 \pi]$ is periodic if and only if $v_{j}(2 \pi)=0$ for 
every $j>1$. Moreover, from the results of Appendix A of [2] one has

$$
\begin{gathered}
K_{1,0}(t)=I_{A}(t), \quad K_{0,1}(t)=I_{B}(t), \\
K_{1,1}(t)=n I_{A B}(t)+(m-n) I_{B A}(t), \\
K_{2,1}(t)=\frac{n m+m(m-1)}{2} I_{A}^{2}(t) I_{B}(t)+(n(n-1)-m(m-1)) I_{A}(t) I_{A B}(t) \\
+(m-1)(m-n) I_{A A B}(t) .
\end{gathered}
$$

In the literature, the first $v_{j}(2 \pi)$ different from zero is known as the Lyapunov constant. We shall compute the Lyapunov constants in order to obtain a Hopf bifurcation of the zero solution. These computations will ensure that conditions (i) and (ii) of Lemma 3.1 hold.

The second problem is to check whether (iii) holds. To this end, we shall use the following necessary condition for the existence of a centre.

Lemma 3.3 ([8]). Take A,B trigonometric polynomials and consider the one-parameter family of differential equations

$$
x^{\prime}=\lambda A(t) x^{n}+B(t) x^{m}, \quad \lambda \in \mathbb{R}, n \neq m, n, m \geq 2 .
$$

If (3.5) has a centre at $x(t) \equiv 0$ for every $\lambda$, then

$$
\int_{0}^{2 \pi} A(t) I_{B}(t)^{j} d t=0 \quad \text { for every } j \in \mathbb{Z}^{+} .
$$

The reciprocal of the above lemma is false. Nevertheless, there is a very strong relationship between the centre problem and the so-called Moment Problem which consists of, given $B(t)$, obtaining all functions $A(t)$ such that

$$
\int_{0}^{2 \pi} A(t) I_{B}(t)^{j} d t=0, \quad \text { for every } j \in \mathbb{Z}^{+} .
$$

This problem has been completely solved for $A(t), B(t)$ polynomials, see [19], and is being studied for the trigonometric case.

The relationship between the aforementioned two problems is the following. Let $u(t, x, \lambda)$ be the solution of Eq. (3.5) such that $u(0, x, \lambda)=x$. The infinitesimal centre condition of Eq. (3.5) at $\lambda=0$, that is, Eq. (3.5) has a centre for $\lambda=0$ and for any solution of $(3.5), \frac{\partial u}{\partial \lambda}(2 \pi, x, 0)=0$, is equivalent to the moment equations $\int_{0}^{2 \pi} A(t) I_{B}(t)^{j} d t=0$, for every $j \in \mathbb{Z}^{+}$. (See $[8$, Theorem 2.1]). However, as was noted above, it is not true that if the functions $A$ and $B$ are such that all the moment equations vanish then Eq. (3.5) has a centre at the origin for every $\lambda$ - it has a centre only at "first order of $\lambda$ ".

Nonetheless, the moment equations will be very useful to prove that an equation of type (3.5) has no centre at the origin. It will be enough to prove that there exists a $j \in \mathbb{Z}^{+}$such that the $j$ moment equation is not zero. In order to find such a $j$, we prove the following result.

Lemma 3.4. Let $A, B$ be analytical functions on the interval $\left[t_{1}, t_{2}\right]$ such that $\left|\int_{t_{1}}^{t} B\right|$, has a unique global maximum at a point $t^{*}$. If $A$ has no changes 
of sign in a neighbourhood of $t^{*}$ and is not identically null then there exists $j \in \mathbb{N}$ such that

$$
\int_{t_{1}}^{t_{2}} A(t)\left(\int_{t_{1}}^{t} B(s) d s\right)^{j} d t \neq 0
$$

Proof. Let $I_{B, t_{1}}(t)=\int_{t_{1}}^{t} B$. Assume that $A(t)$ is positive in a neighbourhood $U$ of $t^{*}$, and that $t^{*}$ is a maximum of $I_{B, t_{1}}(t)$, with the other cases being analogous. We prove that there exists $M<I_{B, t_{1}}\left(t^{*}\right)$ such that $V=I_{B, t_{1}}^{-1}((M, \infty)) \subset U$. Indeed, assume that there exist $M_{k}<I_{B, t_{1}}\left(t^{*}\right)$ and $t_{k} \in I_{B, t_{1}}^{-1}\left(\left(M_{k}, \infty\right)\right)$ such that $M_{k} \rightarrow I_{B, t_{1}}\left(t^{*}\right)$ and $t_{k} \notin U$. One may assume that $t_{k} \rightarrow t^{* *} \neq t^{*}$. Then $I_{B, t_{1}}\left(t_{k}\right) \rightarrow I_{B, t_{1}}\left(t^{* *}\right)=I_{B, t_{1}}\left(t^{*}\right)$ in contradiction with the uniqueness of the maximum $t^{*}$.

Thus, there exists $V \subseteq U$ such that $A(t) \geq 0$ and $I_{B, t_{1}}(t)>M \geq\left|I_{B, t_{1}}(s)\right|$ for all $t \in V, s \notin V$. Consequently

$$
\int_{t_{1}}^{t_{2}} A(t) \frac{I_{B, t_{1}}^{j}(t)}{M^{j}} d t \rightarrow+\infty \quad \text { as } j \rightarrow \infty .
$$

Therefore, there exists $j$ such that

$$
\int_{t_{1}}^{t_{2}} A(t) I_{B, t_{1}}^{j}(t) d t=M^{-j} \int_{t_{1}}^{t_{2}} A(t) \frac{I_{B, t_{1}}^{j}(t)}{M^{j}} d t \neq 0 .
$$

\section{LOWER BOUNDS ON THE NUMBER OF LIMIT CYCLES}

To conclude the proof of Theorem 1.2, one needs to show that, in any of the cases not included in Propositions 2.2 or 2.3, there exists a limit cycle different from $x(t) \equiv 0$.

Proposition 4.1. Assume that (1.2) does not satisfy the sufficient conditions in Propositions 2.2 and 2.3. Then, reordering $\left\{A_{1}, \ldots, A_{k}\right\}$ if necessary, one of the following cases holds:

(I.1) $A_{1}, A_{2} \in \mathcal{E}$.

(I.2) $A_{1}, B \in \mathcal{E}$.

(I.3) $A_{1} \in \mathcal{E}, A_{2} \in \mathcal{C}$ (resp. $\mathcal{S}$ ), and $B \in \mathcal{S}$ (resp. $\mathcal{C}$ ).

(I.4) $A_{1} \in \mathcal{E}, A_{2} \in \mathcal{S}, A_{3} \in \mathcal{C}$, and $B \in \mathcal{O}$.

(I.5) $A_{1}, A_{2} \in \mathcal{C}$ (resp. $\mathcal{S}$ ) and $B \in \mathcal{S}$ (resp. $\mathcal{C}$ ).

(I.6) $A_{1} \in \mathcal{S}, A_{2} \in \mathcal{C}, A_{3} \in \mathcal{S} \cup \mathcal{C}$, and $B \in \mathcal{O}$.

Proof. If equation (1.2) does not satisfy the sufficient conditions in Propositions 2.2 and 2.3 then, for any reordering $\left\{A_{1}, \ldots, A_{k}\right\}$, none of the following assumptions hold:

(N.1) $A_{1}, \ldots, A_{k}, B \in \mathcal{S} \cup \mathcal{O}$, or $A_{1}, \ldots, A_{k}, B \in \mathcal{C} \cup \mathcal{O}$.

(N.2) $A_{1} \in \mathcal{E}$ and (1.2) for $a_{1}=0$ has a centre at $x(t) \equiv 0$.

(N.3) $B \in \mathcal{E}$ and (1.2) for $b=0$ has a centre at $x(t) \equiv 0$.

(N.4) $A_{1} \in \mathcal{S}, A_{2}, \ldots, A_{k} \in \mathcal{C} \cup \mathcal{O}$, and $B \in \mathcal{C}$. 
(N.5) $A_{1} \in \mathcal{C}, A_{2}, \ldots, A_{k} \in \mathcal{S} \cup \mathcal{O}$, and $B \in \mathcal{S}$.

(N.6) $A_{1} \in \mathcal{S}, A_{2} \in \mathcal{C}, A_{3}, \ldots, A_{k} \in \mathcal{O}$, and $B \in \mathcal{O}$.

If one of the previous conditions holds then there is at most one function among $\left\{A_{1}, \ldots, A_{k}, B\right\}$ belonging to $\mathcal{E}$. Thus, if there exist two functions among $\left\{A_{1}, \ldots, A_{k}, B\right\}$ belonging to $\mathcal{E}$, then none of (N.1), (N.2), ., (N.6) hold. Therefore, after a reordering, either (I.1) or (I.2) holds.

Now, assume that only one of the functions $A_{1}, \ldots, A_{k}, B$ belongs to $\mathcal{E}$. If $B \in \mathcal{E}$ and (1.2) for $b=0$ has no centre at $x(t) \equiv 0$ then $A_{l} \in \mathcal{E}$ for some $1 \leq l \leq k$, in contradiction with our hypothesis. If $A_{1} \in \mathcal{E}$ and (1.2) for $a_{1}=0$ has no centre at $x(t) \equiv 0$ then either (I.3) or (I.4) holds.

Finally, assume that none of $\left\{A_{1}, \ldots, A_{k}, B\right\}$ belong to $\mathcal{E}$. We shall distinguish two cases: when $B \in \mathcal{C}$ (resp. $B \in \mathcal{S}$ ), and when $B \in \mathcal{O}$. If $B \in \mathcal{C}$ (resp. $B \in \mathcal{S}$ ), since neither (N.1) nor (N.4) hold, then at least two of the functions $A_{1}, \ldots, A_{k}$ belong to $\mathcal{S}$ (resp. $\mathcal{C}$ ), and, after reordering, (I.5) holds. If $B \in \mathcal{O}$, since neither (N.1) nor (N.6) hold, then at least two of the functions $A_{1}, \ldots, A_{k}$ belong to $\mathcal{S}$ and one to $\mathcal{C}$, or at least two of the functions $A_{1}, \ldots, A_{k}$ belong to $\mathcal{C}$ and one to $\mathcal{S}$. In any case, after reordering, (I.6) holds.

Now we shall study the Hilbert number for (1.2), when the monomials satisfy one of the conditions (I.1)-(I.6).

Proposition 4.2. Assume that one of (I.1)-(I.6) holds. Then there exist coefficients $a_{1}, \ldots, a_{k}, b$ such that (1.2) has at least one limit cycle different from $x(t) \equiv 0$. Therefore (1.2) has $\mathcal{H} \geq 2$.

Proof. Assume that (1.2) satisfies one of (I.1)-(I.6).

If (I.2) or (I.3) holds, then Theorem 3.3 of [2] proves that there exist $a_{1}, b \in \mathbb{R}$ or $a_{1}, a_{2}, a_{3} \in \mathbb{R}$ such that (1.2) has one limit cycle different from $x(t) \equiv 0$.

If (I.1) holds and $B \in \mathcal{S}$ (the case $B \in \mathcal{C}$ follows after the change of variable $t \rightarrow \pi / 2-t$ ), by Lemma 3.2 , for every $j>1, v_{j}(t) \equiv 0$ except for $j=k_{1} n+k_{2} m-\left(k_{1}+k_{2}-1\right)$. Moreover, since $B$ is odd, (1.2) has a centre at $x(t) \equiv 0$ for $a_{1}=a_{2}=0$. Then $K_{0, k_{2}}(2 \pi)=0$ for every $k_{2} \in \mathbb{N}$. Consequently, the lowest $j=k_{1} n+k_{2} m-\left(k_{1}+k_{2}-1\right)$ such that $v_{j}(2 \pi) \neq 0$ must have $k_{1}>0$. Indeed, this is obtained for $k_{1}=1$ and $k_{2}=0$ since

$$
v_{n}(2 \pi)=a_{1} I_{A_{1}}(2 \pi)+a_{2} I_{A_{2}}(2 \pi),
$$

and $I_{A_{1}}(2 \pi), I_{A_{2}}(2 \pi)>0$. Then $v_{n}(2 \pi)=0$ if and only if

$$
a_{2}=-a_{1} \frac{I_{A_{1}}(2 \pi)}{I_{A_{2}}(2 \pi)} .
$$

By Lemma 3.1 with $j^{*}=n$, it is sufficient to prove that (1.2) has no centre at $x(t) \equiv 0$ for some $a_{1},-a_{1} \frac{I_{A_{1}}(2 \pi)}{I_{A_{2}}(2 \pi)}$, and $b$. Take $b=1, \lambda=a_{1}$ in 
equation (3.5), and $A=A_{1}-\frac{I_{A_{1}}(2 \pi)}{I_{A_{2}}(2 \pi)} A_{2}$. By Lemma 3.3, one only needs to prove that there exists $j$ such that

$$
\int_{0}^{2 \pi} A(t) I_{B}^{j}(t) d t \neq 0 .
$$

Since $I_{B}(t)$ is increasing in $(0, \pi)$ and decreasing in $(\pi, 2 \pi),\left|I_{B}(t)\right|$ has a unique maximum at $t=\pi$. Since $A(t-\pi)$ is even, it has no changes of sign in a neighbourhood of $t=\pi$. By Lemma 3.4, there exists $j$ such that (4.6) holds. Therefore (1.2) has no centre at $x(t) \equiv 0$ for some $a_{1} \in \mathbb{R}$.

If $B \in \mathcal{O}$, the proof follows as before, now just taking into account that $A(t-\pi)$ and $I_{B}(t-\pi)$ are even, and therefore

$$
\int_{0}^{2 \pi} A(t) I_{B}^{j}(t) d t=2 \int_{0}^{\pi} A(t) I_{B}^{j}(t) d t .
$$

Now $I_{B}(t)$ has its unique maximum for $t \in(0, \pi)$ at $t=\pi / 2$, and $A(t-\pi / 2)$ is even and hence has no changes of sign in a neighbourhood of $t=\pi / 2$.

If (I.4) holds, arguing as in (I.1), since $I_{A_{1}}(2 \pi)>0$, the first $v_{j}(2 \pi)$ that can be different from zero is

$$
v_{n}(2 \pi)=a_{1} I_{A_{1}}(2 \pi) .
$$

For $a_{1}=0, a_{2} \neq 0, a_{3} \neq 0$ and $b \neq 0$, applying Proposition 2.3 one obtains that $x(t) \equiv 0$ is the only limit cycle of $(1.2)$, then it is not a centre. The proof follows by Lemma 3.1 .

Assume that (I.5) holds and that $A_{1}, A_{2} \in \mathcal{C}$, and $B \in \mathcal{S}$. The other case follows by the change of variable $t \rightarrow \pi / 2-t$.

Now, if $a_{1}=a_{2}=0$ or $b=0$ then (1.2) has a centre at $x(t) \equiv 0$. Then $K_{k_{1}, 0}(2 \pi)=0$ and $K_{0, k_{2}}(2 \pi)=0$. Consequently, the first $v_{j}(2 \pi)$ that can be different from zero is

$$
v_{n+m-1}(2 \pi)=a_{1} b(n-m) I_{A_{1} B}(2 \pi)+a_{2} b(n-m) I_{A_{2} B}(2 \pi) .
$$

By integration by parts, $I_{A_{1} B}(2 \pi)+I_{B A_{1}}(2 \pi)=I_{A_{1}}(2 \pi) I_{B}(2 \pi)$. As $A_{1} \in \mathcal{C}$, $I_{A_{1}}(2 \pi)=0$, and $I_{A_{1} B}(2 \pi)=-I_{B A_{1}}(2 \pi)$. Since $I_{A_{1}}(t), B(t)$ are positive in $(0, \pi)$ and negative in $(\pi, 2 \pi), I_{B A_{1}}(2 \pi)>0$. Analogously, $I_{A_{2} B}(2 \pi)<0$.

Fix $b>0$. By Lemma 3.1 for $j^{*}=n+m-1$, one only needs to prove that (1.2) has no centre at $x(t) \equiv 0$ for some $a_{1}, a_{2}$ such that

$$
a_{1}=-a_{2} \frac{I_{A_{2} B}(2 \pi)}{I_{A_{1} B}(2 \pi)} .
$$

Now, taking into account that $I_{B}(t)$ has a unique maximum at $t=\pi$ and that $A_{1}(t-\pi), A_{2}(t-\pi)$ are even, thus $A(t)=a_{1} A_{1}(t)+a_{2} A_{1}(t)$ has no changes of sign in a neighbourhood of $\pi$, the proof follows by Lemma 3.4 and Lemma 3.3. 
Assume that (I.6) holds and that $A_{1} \in \mathcal{S}, A_{2} \in \mathcal{C}, A_{3} \in \mathcal{C}, B \in \mathcal{O}$. The other case follows by the change of variable $t \rightarrow \pi / 2-t$.

Let $A=a_{1} A_{1}+a_{2} A_{2}+a_{3} A_{3}$. If $b=0$ then (1.2) has a centre, therefore, from Lemma 3.2 one obtains $K_{k_{1}, 0}(2 \pi)=0$. Analogously, if $a_{k}=0$ for every $k$ then $(1.2)$ has a center and then $K_{0, k_{2}}(2 \pi)=0$. Moreover, by the symmetries of $A$ and $B$, one obtains $I_{A_{i} B}(2 \pi)=0$, then $K_{1,1}(2 \pi)=0$. Thus the first $v_{j}(2 \pi)$ that can be different from zero is

$$
v_{2 n+m-2}(2 \pi)=(m-1)(m-n) I_{A A B}(2 \pi) .
$$

One has

$$
I_{A A B}(2 \pi)=\sum_{i, j=1}^{3} a_{i} a_{j} I_{A_{i} A_{j} B}(2 \pi) .
$$

By symmetry, $I_{A_{i} A_{i} B}(2 \pi)=0$ for $i=1,2,3$. Then

$$
\begin{aligned}
I_{A A B}(2 \pi) & =a_{1} a_{2} b\left(I_{A_{1} A_{2} B}(2 \pi)+I_{A_{2} A_{1} B}(2 \pi)\right)+a_{1} a_{2} b\left(I_{A_{1} A_{3} B}(2 \pi)+I_{A_{3} A_{1} B}(2 \pi)\right) \\
& =a_{1} a_{2} b \int_{0}^{2 \pi} I_{A_{1}}(t) I_{A_{2}}(t) B(t) d t+a_{1} a_{3} b \int_{0}^{2 \pi} I_{A_{1}}(t) I_{A_{3}}(t) B(t) d t .
\end{aligned}
$$

Therefore

$$
\begin{aligned}
& v_{2 n+m-2}(2 \pi) \\
= & (m-1)(m-n) a_{1} b\left(\int_{0}^{2 \pi}\left(a_{2} I_{A_{1}}(t) I_{A_{2}}(t) B(t)+a_{3} I_{A_{1}}(t) I_{A_{3}}(t) B(t)\right) d t\right) .
\end{aligned}
$$

Set

$$
\mu=\frac{\int_{0}^{2 \pi} B(t) I_{A_{1}}(t) I_{A_{2}}(t) d t}{\int_{0}^{2 \pi} B(t) I_{A_{1}}(t) I_{A_{3}}(t) d t} .
$$

Since $B(t) I_{A_{1}}(t) I_{A_{2}}(t)$ is even and

$$
0<B(t) I_{A_{1}}(t) I_{A_{2}}(t)<-B(\pi-t) I_{A_{1}}(\pi-t) I_{A_{2}}(\pi-t),
$$

one has that

$$
\int_{0}^{2 \pi} B(t) I_{A_{1}}(t) I_{A_{2}}(t) d t=2 \int_{0}^{\pi} B(t) I_{A_{1}}(t) I_{A_{2}}(t) d t<0 .
$$

Analogously, $\int_{0}^{2 \pi} B(t) I_{A_{1}}(t) I_{A_{3}}(t) d t<0$, and therefore $\mu>0$.

Let $a_{3}=-\mu a_{2}$. If we prove that $x(t) \equiv 0$ with coefficients $a_{1}, a_{2},-\mu a_{2}, b \neq$ 0 is not a centre, then from Lemma 3.1 we will obtain the existence of a positive limit cycle.

Denote

$$
F(t)=a_{2}\left(A_{2}(t)-\mu A_{3}(t)\right)=\sin ^{2 i}(t) \cos ^{2 j+1}(t) G(t),
$$

where $i=\min \left(i_{1}, i_{2}\right), j=\min \left(j_{1}, j_{2}\right)$, and

$$
G(t)=a_{2}\left(\sin ^{2\left(i_{2}-i\right)}(t) \cos ^{2\left(j_{2}-j\right)}(t)-\mu \sin ^{2\left(i_{3}-i\right)}(t) \cos ^{2\left(j_{3}-j\right)}(t)\right) .
$$

Let us prove that we may assume that $G(k \pi / 2) \neq 0$ for every $k \in \mathbb{Z}$. If $G(k \pi / 2)=0$ for certain $k \in \mathbb{Z}$ then $\mu=1$ and either $i_{2}-i=i_{3}-i=0$ or 
$j_{2}-j=j_{3}-j=0$. In any case $F(t)$ has a definite sign at $(\pi / 2,3 \pi / 2)$. By

[7, Theorem 2.4], one obtains that (1.2) is not a centre.

One has the following possibilities depending on whether $i=i_{1}, i=i_{2}$, $j=j_{1}$, or $j=j_{2}$ :

$$
\begin{aligned}
& A(t)=\sin ^{2 i} t \cos ^{2 j+1} t\left(a_{1} \sin ^{2\left(i_{1}-i\right)+1} t \cos ^{2\left(j_{1}-j\right)-1} t+G(t)\right), \quad \text { or } \\
& A(t)=\sin ^{2 i} t \cos ^{2 j_{1}} t\left(a_{1} \sin ^{2\left(i_{1}-i\right)+1} t+\cos ^{2\left(j-j_{1}\right)+1} t G(t)\right),
\end{aligned}
$$

$$
\begin{aligned}
& A(t)=\sin ^{2 i_{1}+1}(t) \cos ^{2 j_{1}}(t)\left(a_{1}+\sin ^{2\left(i-i_{1}\right)-1} t \cos ^{2\left(j-j_{1}\right)+1} t G(t)\right), \quad \text { or } \\
& A(t)=\sin ^{2 i_{1}+1}(t) \cos ^{2 j+1}(t)\left(a_{1} \cos ^{2\left(j_{1}-j\right)-1}(t)+\sin ^{2\left(i-i_{1}\right)-1}(t) G(t)\right),
\end{aligned}
$$

where all the exponents are chosen to be positive.

We shall apply Lemma 3.3 where the roles of $A$ and $B$ have been interchanged.

Assume (4.7) holds. For $a_{2}=0,\left|I_{A}(t)\right|$ has its unique maximum at $t=\pi$. Let $a_{2}$ be small enough. Since $G(\pi) \neq 0$, one has that $\frac{d^{2 i} A}{d t^{2 i}}(\pi) \neq 0$ and $I_{A}$ has no extremum point at $\pi$. Therefore $\left|I_{A}(t)\right|$ has its unique global maximum at $t^{*} \neq \pi$ close to $\pi$.

Assume now that (4.8) holds. Denote by $t_{k} \in(0,2 \pi), k=1, \ldots, r$, the global maxima of $I_{F}(t)$. Since $I_{F}(t)$ is odd and $2 \pi$-periodic, $\left\{2 \pi-t_{k}\right\}_{1 \leq k \leq r}$ are the global minima. Moreover, $t_{k} \neq \pi$. Let $t^{*}$ be the $t_{k}$ closest to $\pi$. There are two possibilities for $t^{*}$ :

First, suppose that $t^{*} \notin\{\pi / 2,3 \pi / 2\}$. Now, since for any $a_{1}>0, a_{1} I_{A_{1}}(t)$ is positive, strictly increasing in $(0, \pi)$ and strictly decreasing in $(\pi, 2 \pi)$, then for $a_{1}>0$ small enough, $\left|I_{A}(t)\right|$ has a unique global maximum at $\bar{t} \in(0,2 \pi)$ (close to $t^{*}$ ), such that $\bar{t} \notin\{\pi / 2, \pi, 3 \pi / 2\}$. One concludes by Lemma 3.4 and Lemma 3.3.

Second, suppose that $t^{*}=\pi / 2$ (the argument is analogous for $t^{*}=3 \pi / 2$ ). Note that then $I_{F}(t)$ has a global minimum at $3 \pi / 2$. Since $I_{A_{1}}(t)$ is positive and increasing for $t \in(0, \pi / 2)$, if one takes $a_{1}>0$ small enough then $\left|I_{A}(t)\right|$ has a unique maximum at a certain $\bar{t}$ close to $\pi / 2$ (it might be precisely at $\pi / 2)$. Moreover, since $F(-\pi / 2+t)$ is odd $I_{F}(-\pi / 2+t)$ is even. Thus for all $t \in(0, \pi / 2)$, one has

$$
I_{A}(\pi / 2+t)>I_{A}(\pi / 2-t) .
$$

Take $\left|I_{A}(\bar{t})\right|>M>0$ such that $\left|I_{A}(\tilde{t})\right|<M$ for any local extremum $\tilde{t} \neq \bar{t}$ of $I_{A}(t)$. Then the equation $I_{A}(t)=M$ has two solutions $t_{-}, t_{+} \in(0, \pi)$, and $t_{-}<\pi / 2<t_{+}$. Moreover, by the inequality (4.9), $\pi / 2-t_{-}<t_{+}-\pi / 2$, that is, $\pi-t_{-}<t_{+}$. Let

$$
I(j)=\int_{0}^{2 \pi} B(t) I_{A}^{j}(t) d t=M^{j} \int_{0}^{2 \pi} B(t)\left(\frac{I_{A}(t)}{M}\right)^{j} d t=M^{j} \int_{0}^{2 \pi} a(t, j) d t,
$$


where

$$
a(t, j)=B(t)\left(\frac{I_{A}(t)}{M}\right)^{j}
$$

We shall prove that there exists $j \in \mathbb{N}$ such that $I(j) \neq 0$ and conclude by Lemma 3.3. Assume on the contrary that $I(j)=0$ for every $j \in \mathbb{N}$. Then

$$
\int_{0}^{2 \pi} a(t, j) d t=0, \quad \text { for every } j \in \mathbb{N} \text {. }
$$

One has that

$$
\int_{0}^{2 \pi} a(t, j) d t=\int_{\left.[0,2 \pi] \backslash t_{-}, t_{+}\right]} a(t, j) d t+\int_{t_{-}}^{\pi-t_{-}} a(t, j) d t+\int_{\pi-t_{-}}^{t_{+}} a(t, j) d t .
$$

The function $a(t, j)$ is bounded for all $t \notin\left(t_{-}, t_{+}\right), j \in \mathbb{N}$. Assume that $b>0$. Since (4.9), $B \in \mathcal{O}$ and $b>0$, then $a(\pi / 2+t, j)<a(\pi / 2-t, j)$ for all $t \in\left(\pi / 2, \pi-t_{-}\right)$and $j \in \mathbb{N}$. Therefore

$$
\int_{t_{-}}^{\pi-t_{-}} a(t, j) d t<0 .
$$

Finally, since $a(t, j)$ is continuous with respect to $t$ and $a(t, j) \rightarrow-\infty$ as $j \rightarrow+\infty$ for all $t \in\left(\pi-t_{-}, t_{+}\right)$, one has $I(j) / M^{j}=\int_{0}^{2 \pi} a(t, j) d t \rightarrow-\infty$ as $j \rightarrow \infty$, in contradiction with $I(j)=0$ for every $j \in \mathbb{N}$.

\section{REFERENCES}

[1] A. Álvarez, J.L., Bravo, M. Fernández, The number of limit cycles for generalized Abel equations with periodic coefficients of definite sign, Commun. Pure Appl. Anal. 8-5, (2009), 1493-1501.

[2] M.J. Álvarez, J.L. Bravo, M. Fernández, Uniqueness of limit cycles for polynomial first-order differential equations, J. Math. Anal. Appl. 360-1, (2009), 168-189.

[3] M.J. Álvarez, A. Gasull, H. Giacomini, A new uniqueness criterion for the number of periodic orbits of Abel equations, J. Differential Equations 234, (2007), 161-176.

[4] M.A.M. Alwash, Periodic solutions of Abel differential equations, J. Math. Anal. Appl. 329, (2007), 1161-1169.

[5] M.A.M. Alwash, N.G. Lloyd, Nonautonomous equations related to polynomial two dimensional systems, Proc. Roy. Soc.Edinburgh, 105A, (1987), 129-152.

[6] J.L. Bravo, M. Fernández, A. Gasull, Limit cycles for some Abel equations with coefficients without fixed signs, Int. J. Bif. Chaos 19-11, (2009), 389-3876.

[7] J.L. Bravo, J. Torregrosa, Abel-like equations with no periodic solutions, J. Math. Anal. Appl., 342, (2008), 931-942.

[8] M. Briskin, J.P. Françoise, Y. Yomdin, Center conditions II: Parametric and model center problems, Isr. J. Math, 118, (2000), 61-82.

[9] B. Coll, A. Gasull, J. Llibre, Some theorems on the existence, uniqueness, and nonexistence of limit cycles for quadratic systems, J. Differential Equations, 67, (1987), 372-399.

[10] J. Devlin, N.G. Lloyd, J.M. Pearson, Cubic systems and Abel equations, J. Differential Equations, 147, (1998), 435-454.

[11] A. Gasull and A. Guillamon. Limit cycles for generalized Abel equations, Int. J. Bif. Chaos, 16, (2006), 3737-3745.

[12] A. Gasull, J. Llibre, Limit cycles for a class of Abel equations, SIAM J. Math. Anal., 21-5 (1990), 1235-1244. 
[13] A. Gasull, R. Prohens, J. Torregrosa, Limit cycles for rigid cubic systems, J. Math. Anal. Appl. 303, (2005), 391-404.

[14] A. Gasull, J. Torregrosa, Some results on rigid systems, In International Conference on Differential Equations (Equadiff-2003), World Sci. Publ., Hackensack, NJ. (2005), 340-345.

[15] Yu. Ilyashenko, Centennial history of Hilbert's 16th problem, Bull. Amer. Math. Soc., 39, (2002), 301-354.

[16] A. Lins Neto, On the number of solutions of the equation $\frac{d x}{d t}=\sum_{j=0}^{n} a_{j}(t) x^{j}, 0 \leq$ $t \leq 1$, for which $x(0)=x(1)$, Inv. Math. 59, (1980), 67-76.

[17] N.G. Lloyd, A note on the number of limit cycles in certain two-dimensional systems, J. London Math. Soc. 20 (1979), 277-286.

[18] V.A. Pliss, Non local problems of the theory of oscillations, Academic Press, New York, 1966.

[19] F. Pakovich, M Muzychuk, Solution of the polynomial moment problem, Proc. London Math. Soc. (3) 99 (2009), 633-657.

[20] A.A. Panov, The number of periodic solutions of polynomial differential equations, Mathematical Notes 64-5, (1998), 622-628.

Departament de Matemàtiques i Informàtica, Universitat de les Illes Balears, 07122, Palma de Mallorca, Spain

E-mail address: chus.alvarez@uib.es

Departamento de Matematicas, Universidad de Extremadura, 06071 BadaJOZ, SPAIN

E-mail address: trinidad@unex.es

Departamento de Matematicas, Universidad de Extremadura, 06071 BadaJOZ, SPAIN

E-mail address: ghierro@unex.es 